\title{
LUTS: Nicht immer ist der Harntrakt erkrankt
}

B eschwerden des unteren Harntrakts ( „lower urinary tract symptoms“, LUTS) bei Männern werden oft mit einem benignen Prostatasyndrom (BPS) gleichgesetzt, sind aber multifaktoriell. Sie werden nicht nur durch Erkrankungen des Harntrakts ausgelöst, betonte PD Dr. Matthias Oelke, Hannover. Ursache könnten zum Beispiel auch neurologische Erkrankungen, Fremdkörper in der Blase oder Diabetes sein. Auch Art, Qualität und Quantität der Harnwegsbeschwerden erlauben keine Rückschlüsse auf die zugrunde liegende Erkrankung und korrelieren nicht mit der Ausprägung der auslösenden Erkrankung.

In der Diagnostik müssen Symptomatik, Blasenauslassobstruktion („bladder outlet obstruction“, BOO) und eine benigne Prostatavergrößerung (BPE) einzeln abgeklärt werden, da alle drei Komponenten eines BPS stark variieren kön- nen, erklärte Oelke. Obligat sind eine umfassende Anamnese mit Erfassung der Symptomatik mittels Fragebögen und körperliche Untersuchung inklusive digital-rektaler Palpation, Abklärung von neurologischen Erkrankungen, medikamenten-induzierten LUTS, Genitalerkrankungen und Prostatakarzinom. Ferner sind Untersuchungen von Urin (Stix) und Blut (Kreatinin, PSA), Ultraschalluntersuchungen von Nieren, Blase, Prostata und eine Uroflowmetrie erforderlich.

Nicht empfohlen werden initial Endoskopie und Röntgen, fakultativ sind urodynamische Untersuchungen und sonografische Messungen der Detrusordicke. Allerdings sei die Detrusordicke eine der besten Prädiktoren einer BOO, erklärte Oelke. „Je dicker die Blasenwand, desto mehr obstruktive Komponenten“, sagte er.
Ist die Diagnose BPS gesichert, sollte medikamentös behandelt werden. Laut einer europäischen Erhebung von 2010 werden in Deutschland aber nur rund ein Viertel der BPS-Patienten mit einem Alphablocker, $4 \%$ mit einem 5-alphaReduktasehemmer (5-ARI) und $7 \%$ mit einem Phytotherapeutikum behandelt, so PD Dr. Christian Gratzke, München. Die besten Ergebnisse werden bei kombiniertem Einsatz von Alphablocker und 5-ARI erzielt. Bei letzteren sei in Bezug auf die Symptomatik etwas Geduld erforderlich, bis sich die maximale Wirkung entfalte, so Gratzke. Das Risiko eines akuten Harnverhaltes wurde durch Kombitherapie im Vergleich zur Alphablocker-Monotherapie um 70-80 \% verringert.

Roland Fath

\section{Prostatakarzinom: Aktuelles zur HAROW-Studie}

n vielen aktuellen Untersuchungen, zum Beispiel der kürzlich publizierten USamerikanischen PIVOT-Studie, schnitt die Strategie der Active Surveillance (AS) bei Patienten mit lokal begrenztem Prostatakarzinom (PCa) nicht schlechter ab als die Intervention. Dennoch werden in der Praxis zurzeit nur rund $10 \%$ der Patienten mit lokal begrenztem PCa aktiv überwacht, sagte PD Dr. Felix Chun, Hamburg.

Was sind die Gründe? Die Entscheidung, welcher Patient zur Niedrig-RisikoGruppe zählt, ist im Einzelfall nicht immer einfach. Die wichtigsten Indikatoren: ein Gleason-Score $\leq 6$, ein PSA-Wert unter $10 \mathrm{ng} / \mathrm{ml}$, ein Tumorstadium T1a-T2b, nur zwei Stanzen aus der 12-er Prostatabiopsie positiv sowie geringes Tumorvolumen. Anhand von Nomogrammen Risikokalkulatoren, die im Internet verfügbar sind - kann das Progressionsrisiko der Patienten beurteilt werden. Alle nicht signifikanten Tumoren können mithilfe solcher Kalkulatoren richtig eingeordnet werden, erklärte Chun. Das Problem: Bei rund 30\% der Männer gibt es falsch-po- sitive Befunde - sie zählen eigentlich nicht zur Risikogruppe. Ob dies relevant ist, ist unklar.

Unter AS wird in der Regel in drei- bis sechsmonatigen Abständen der PSA-Wert kontrolliert, Folgebiopsien erfolgen nach ein bis zwei Jahren. Eine verspätete Intervention hat nach Ansicht von Dr. Corinna Schäfer, Leipzig, kaum Nachteile. Die Entscheidung zwischen den beiden Strategien bei lokal begrenztem Prostatakarzinom scheint allerdings nicht nur eine Frage der wissenschaftlichen Grundlagen. Es herrscht auch eine Art Glaubenskrieg unter Urologen: Für viele Urologen scheint die AS keine echte Therapieoption zu sein. Die HAROW-Sitzung, auf der neue Daten zur AS vorgestellt wurden, war auf dem Kongress als Nicht-DGUSitzung aus dem Programm praktisch ausgelagert und fand vor weniger als 100 Zuhörern statt.

HAROW ist eine deutsche Versorgungsforschungsstudie in Zusammenarbeit mit der Stiftung Männergesundheit, die vom Gasunternehmen Gazprom finanziell unterstützt wird. Seit 2008 wur- den in die über fünf Jahre laufende Studie 2.848 Patienten mit lokal begrenztem PCa aufgenommen. Rund die Hälfte der Patienten wurde sofort operiert, $15 \%$ wurden aktiv überwacht, weitere $5 \%$ ohne feste Kontrolltermine überwacht („watchful waiting“), berichtete Dietrich Schnell, Berlin. Von den 376 Patienten der ASGruppe ist bisher - bei einem Follow-up von im Schnitt 1-1,5 Jahren - keiner am PCa gestorben und bei 46 Patienten (12\%) wurde AS gestoppt - bei den meisten davon aufgrund eines Progresses. Die Verlaufszeit der Studie ist noch viel zu kurz, um über die AS-Strategie ein Urteil zu fällen, waren sich die Beteiligten einig. Aber die Zukunft der AS und aktuelle Behandlungsempfehlungen bei lokal begrenztem Prostatakarzinom können nur aus der Versorgungsforschung abgeleitet werden, betonte Prof. Lothar Weißbach, Berlin.

Roland Fath

Nicht-DGU-Sitzung „Active Surveillance Eine Alternative für das Niedrigrisiko-

Prostatakarzinom" 